questionnaire in the week preceding the event. 11809 runners completed the survey.

Interventions (or Assessment of Risk Factors) Demographics including age, gender, experience and training history.

Results The average age of respondents was 40.9y (range 18$83 y)$ and $54.1 \%$ were male. $22.3 \%$ of respondents developed a new illness in the 4 weeks prior to the event. Upper respiratory tract infection (URTI) was most common (64.3\%), followed by GI problems (15.4\%) and headache/migraine (14.6\%). 28.5\% of respondents who had been training for $<2$ months developed an acute illness, compared with $19.8 \%$ of those trained for $>6$ months $(p=0.0002)$. Lower average weekly training distance $(22.9 \%$ of those training from $<20$ to 40 miles/week vs. $18.7 \%$ training from 40 to $>50$ miles/ week; $\mathrm{p}<0.05)$ and shorter longest training run $(24.4 \%$ whose longest run was $<20$ miles vs. $19.4 \%$ whose longest training run was $>20$ miles; $\mathrm{p}<0.05$ ) were associated with higher incidence of acute illness. $25.0 \%$ of novice runners (running $<1 \mathrm{y}$ ) developed an acute illness compared to $20.3 \%$ of those who had been running $>10 y(p<0.05)$.

Conclusions Novice runners who train for $<2$ months with low average weekly training mileage were more likely to develop an acute illness during marathon training than more experienced runners. Further research is needed to establish the direction and relationship between these factors before guidance can be issued.

\section{WHAT ARE THE MAIN RISK FACTORS FOR LOWER- EXTREMITY RUNNING-RELATED INJURIES? A RETROSPECTIVE SURVEY-BASED ON 3669 RESPONDENTS}

\footnotetext{
1,2Damien Sanfilippo, ${ }^{1}$ Charlotte Beaudart, ${ }^{1}$ Olivier Bruyère, ${ }^{1,2}$ Jean-François Kaux, ${ }^{2}$ Géraldine Martens. 'University and University Hospital of Liège, Liège, Belgium; ${ }^{2}$ ReFORM, Research Centre for the Prevention of Injury and IIIness and the Protection of Athletes, Liège, Belgium
}

\subsection{6/bjsports-2021-IOC.303}

Background Many studies attempt to identify the risk factors for running-related injuries (RRI), but these are not yet well established.

Objectives To investigate the risk factors of RRI.

Design Retrospective online survey-based study among population of runners injured and non-injured.

Setting Leisure road and trail runners

Patients Participants have to be at least 18 years old and have to practice running at least for 12 months. 3669 runners reported information which were included for statistical analysis.

Assessment of Risk Factors The online survey included 41 questions with five main categories: personal characteristics daily lifestyle- training and running characteristics - practice of others sports activities and prevention habits.

Main Outcome Measurements Occurrence of running-related injury over the last 12 months.

Results Amongst the 3669 runners, 1852 (50.5\%) reported at least one injury over the last 12 months. Overuse injury were largely represented (60.6\%). The variables associated with RRI which remained significant in the fully-adjusted model were: previous injury $(\mathrm{OR}=1.63$, IC 95\% $=1.42-1.47)$, competition running $(\mathrm{OR}=1.62$, IC $95 \%=1.26-2.09)$, more than 2 hours running per week $(\mathrm{OR}=1.30$, IC 95\%= $1.03-1.65)$, mileage $(>20 \mathrm{~km} /$ week $)(\mathrm{OR}=1.25$, IC $95 \%=1.01-1.55)$ and speed training $(\mathrm{OR}=1.23$, IC 95\%=1.06-1.48). Univariate analysis revealed other variables associated with more RRI: Trail runners (versus road runners, $\mathrm{p}<0.001$ ), men (versus women, $\mathrm{p}<0.001)$, higher age $(\mathrm{p}<0.001)$, $>2$ running session/week $(\mathrm{p}<0.001)$.

Conclusions Previous injury remains the most relevant RRI risk factor according to the current study and previous data. Many training characteristics seem to be involved but still have to be confirmed in view of conflicting data in literature. Trail runners are more at risk of RRI. Further research would help to understand better RRI and to prevent them.

\section{EPIDEMIOLOGY OF INJURY AND ILLNESS AMONG TRAIL RUNNERS: A SYSTEMATIC REVIEW}

${ }^{1}$ Carel Viljoen, ${ }^{2}$ Christa Janse van Rensburg, ${ }^{3}$ Evert Verhagen, 4,5,6,7 Willem van Mechelen, ${ }^{8}$ Rita Tomas, ${ }^{10}$ Marlene Schoeman, ${ }^{9}$ Susan Scheepers, ${ }^{1}$ Elzette Korkie. ${ }^{1}$ Department of Physiotherapy, Faculty of Health Sciences, University of Pretoria and Sport, Exercise Medicine and Lifestyle Institute (SEMLI), Pretoria, South Africa; ${ }^{2}$ Sport, Exercise Medicine and Lifestyle Institute (SEMLI) and Section Sports Medicine Faculty of Health Sciences, University of Pretoria, Pretoria, South Africa; ${ }^{3}$ Amsterdam Collaboration for Health and Safety in Sports, Department of Public and Occupational Health, Amsterdam Movement Sciences, VU University Medical Center, Amsterdam, Netherlands; ${ }^{4}$ Amsterdam Collaboration for Health and Safety in Sports, Department of Public and Occupational Health, Amsterdam Movement Sciences, VU University Medical Center, Amsterdam, The Netherlands, Amsterdam, Netherlands; ${ }^{5}$ School of Human Movement and Nutrition Sciences, Faculty of Health and Behavioural Sciences, University of Queensland, Brisbane, Australia; ${ }^{6}$ Division of Exercise Science and Sports Medicine (ESSM), Department of Human Biology, Faculty of Health Sciences, University of Cape Town, Cape Town, South Africa; ' School of Public Health, Physiotherapy and Population Sciences, University College Dublin, Dublin, Ireland; ${ }^{8}$ Portugal Football School, Portuguese Football Federation, Lisbon, Portugal; ${ }^{9}$ Department of Library Services, Faculty of Health Sciences, University of Pretoria, Pretoria, South Africa; ${ }^{10}$ None, Pretoria, South Africa

\subsection{6/bjsports-2021-IOC.304}

Background Trail running (TR) is characterised by uneven varying running surfaces, with large elevation gains/losses. Therefore, the injury and illness profiles of TR may differ compared to road running. Limited information is available on injury and illness among trail runners (TRs) to help develop interventions towards injury prevention.

Objective Systematically review data on TR injury and illness.

Design Systematic review.

Setting MEDLINE Ovid, PubMed, Scopus, SportsDiscus, CINAHL, Health Source: Nursing/Academic, Health Source: Consumer Ed. and Cochrane were searched from inception to February 2019. Methodological quality was assessed using an adapted Downs and Black assessment tool.

Patients (or Participants) N/A (systematic review abstract).

Interventions (or Assessment of Risk Factors) Studies were included if they investigated injury and/or illness among TRs participating in training/racing and full-text available in English/French. Studies were excluded on biomarkers of injury/illness in the absence of participants reporting injury/illness, or if no clear evidence was found of investigating TR.

Main Outcome Measurements TR injury (incidence, prevalence, anatomical site, tissue type, specific diagnosis, severity) and illness (incidence, prevalence, symptoms, specific diagnosis, body system, severity).

Results Fourteen studies with 3094 participants were included. Six studies investigated injuries and illnesses, 3 studies investigated only injuries and 5 studies only illnesses. Twelve studies investigated race-related injury and/or illness and 2 studies 